\title{
Open-Loop Electrowetting Actuation with Micro-Stepping
}

\author{
Qi Ni ${ }^{1}$, Daniel E. Capecci' ${ }^{1}$, Nathan B. Crane ${ }^{1, a)}$ \\ ${ }^{1}$ Departement of Mechanical Engineering, University of South Florida, Tampa, U.S.A
}

Microfluidic-driven mechanical actuation opens new possibilities for positioning and manipulating delicate small components. However, existing microfluidic actuation methods are not well-suited to positioning with high resolution. This paper reports a method for precise, open-loop control of droplet position in finite steps by varying the duty cycle of the input signal in electrowetting actuation. When wetted to a solid object, both the droplet and the solid can be actuated. Unlike conventional electrowetting actuation methods, positioning resolution in our proposed method can be much smaller than the size of the underlying electrodes without requiring closed loop feedback control system. Using a leaky dielectric coating, the electrode/electrolyte combination in our device acts as a simple diode by blocking current in one direction and conducting in the other. Each duty cycle of the applied AC square wave corresponds to a unique position on the electrode. The position duty cycle relationship is found to be nonlinear but symmetric about the center of the electrodes. This approach provides a method for improving open-loop positioning resolution without adding more electrodes. Positioning is within $0.2 \mathrm{~mm}(<2.5$ $\%$ of the droplet diameter $)$ of the idealized model and repeatability is $<0.07 \mathrm{~mm}(<0.8 \%$ of the droplet diameter $)$.

\section{Introduction}

Many techniques are available for microscale actuation including electrostatic, piezoelectric, thermal, and magnetic [1,2]. However, these actuation methods are characterized by high stiffness and low displacement. Additionally, the actuator size is typically many times larger than the range of motion that they provide. While piezoelectric and thermal actuators generate large forces with high precision, the high stiffness makes it difficult to handle delicate components without damage. Fluidic actuation provides a potential alternative method with inherent compliance that will protect delicate components [3, 4]. Fluidic actuation has been accomplished by viscous forces due to flows in a bulk liquid [5], but surface tension-based manipulation has proven especially effective in microscale bonding for self-assembly and other applications $[6,7]$. Electrowetting is a leading way of manipulating fluids with surface effects.

Electrowetting is a phenomenon in which a fluid interface is deformed by an electric field between an electrode and a fluid. It is commonly implemented with a dielectric covering the electrode to reduce reactions. This is termed electrowetting on dielectric (EWOD). While most applications focus on the movement of droplets or liquid interfaces [8], droplets have also been 
used to perform out of plane mechanical actuation as an alternative to electrostatic actuation [9] as well as both linear and rotational in-plane motions $[10,11]$. Continulus motion similar to-ectromechanical motors was demonstrated using metallic droplets and using embedded diodes.Continuous motion similar to electromechanical motors was demonstrated using metallic droplets [10] and using embedded diodes [12]. However, the majority of EWOD actuation is done in open-loop stepped systems analogous to a stepper motor. Each electrode acts as a pole and the droplet covers it when energized. Researchers have demonstrated a micro conveyor system in which electrowetting droplets move a solid reaction platform [13].

EWOD-based capillary actuators are much more compact than conventional (mechanical, thermal, piezoelectric) microactuators _ _ requiring only the droplet and a substrate. Actuation parameters such as maximum travel and force can be tailored to specific application and cover a variety of length scales with droplets from microns to millimeters and unlimited displacements. Due to the inherent compliance introduced by the fluidic interface, delicate parts can be handled with little risk of damage [14]. In order to maximize the actuation force and speed of EWOD droplets, grounding the droplet is usually favored over a floating configuration $[15,16]$. However, if a grounded droplet is used to carry solid objects, the droplet must be grounded from below. This increases the routing challenges relative to a ground-from-above scheme typically used in pure fluidic actuation $[17,18]$.

The key limitation in EWOD stepping actuation is that current methods do not provide both large travel distance and precise positioning. In stepped actuation, displacement larger than the droplet diametersdiameter requires that the electrodes must be smaller than the droplet's contact area on the substrate. Control algorithms must turn on/off a large number of electrodes to control the motion [19-21]. When large travel distance or two-dimensional motion is required, the number of electrodes can rapidly increase, generating significant wiring and control challenges. While smaller electrodes (and thus smaller droplets) increases position resolution, it reduces the vertical load capacity and the horizontal actuation force. The reduced actuation force also reduces the actuation speed.

Finer motion has been demonstrated by hybrid methods that implement feedback control to move the droplet as small as 1/5 of the electrode [22]. However, this also imposes additional sensing and control requirements that increase the system complexity. Electrowetting actuation systems would benefit from open-loop positioning capability with sub-electrode resolution analogous to electromechanical stepper motors with micro-stepping, which divides each pole into additional segments and provides higher resolution while reducing control complexity.

Previously, we have reported on a bi-directional electrowetting scheme which induces a polarity dependence [23] in the 
electrode pads to continuously move a droplet [12]. Using a thin dielectric with inherent defects, the electrolyte/electrode interface rapidly forms and dissolves a thin oxide on metals such as aluminum and titanium for anodic and cathodic polarizations respectively. The electrolyte/metal interface is effectively an electrochemical diode. By using electrodes pairs with one anodic and one cathodic polarizations, current is limited to achieve long life[24]. This paper shows how $A C$ signals applied to a diode-based actuation to generate a series of stable equilibrium points for the droplet on a single electrode pair. The location of the equilibrium varies with the duty cycle of the input waveform to move droplets in steps that are a fraction of an electrode in length.

Fan et al. [23] demonstrated open loop actuation of a droplet across a substrate using just a square wave. This was accomplished due to a small asymmetry in the contact angle $\left(\sim 10^{\circ}\right)$ between positive and negative electrowetting potentials when using particular dielectric/hydrophobic material combinations. This asymmetry is utilized to create a polarity-dependent actuation method with a small floating droplet that simplifies the electrical actuation control so that a square wave generates $\underline{\text { continuous actuation. However, asymmetrical electrodes are needed for continuous droplet pumping and the pumping direction }}$ was limited. It would be necessary to develop additional actuation mechanism to further improve the actuation directionality.

We have reported on a bi-directional electrowetting scheme which induces a polarity dependence [24] in the EW response through the use of a passivating conductive layer (aluminum, titanium) and an adaption that uses only two electrode pads to continuously move a droplet with a DC voltage input [12]. Using a thin dielectric with inherent defects, the electrolyte/electrode $\underline{\text { interface rapidly forms and dissolves a thin oxide on metals such as aluminum and titanium for anodic and cathodic polarization }}$ respectively. The electrolyte/metal interface is effectively an electrochemical diode. By using electrodes pairs with one anodic and one cathodic polarization, current is limited to achieve long life [25]. This paper shows how AC signals are applied to a diode-based actuation to generate a series of stable equilibrium points for the droplet on a single electrode pair. The location of the equilibrium varies with the duty cycle of the input waveform to move droplets in steps that are a fraction of an electrode in length.

The droplet response is dependent on the frequency of the input waveform. At low frequencies, the droplet will follow the voltage and respond to the instantaneous voltage. This can be used to achieve continuous and oscillatory actuation as in the work of Fan et al. [23]. However, at higher frequencies, the droplet response is a function of the time-averaged voltage. At still higher frequencies, the droplet's impendence becomes significant and electrowetting response declines and liquid dielectrophoresis (DEP) dominates [26]. This work utilizes AC signals at sufficiently high frequency that the droplet responds to the time averaged or RMS voltage. The boundaries between different regimes depends on the droplet size, electrowetting number, and electrolyte conductivity. The test frequency was chosen by increasing the signal frequency well past the point at 


\section{all testing.}

Although both the continuous pumping and step actuation methods are based on electrochemical diodes, the droplet in the continuous actuation system was actuated as a linear motor with constant voltage input [12]. In this paper, asymmetric AC signals are used to create a stepper motor with microstepping. The voltage over each electrode depends on the relative capacitance of the two sides of the circuit. For each duty cycle, there is a unique position at which the net force on the droplet is zero because the voltage imbalance arising from the capacitance difference is offset by longer application time. Circuit simulation with idealized elements is used to understand the voltage response of the system and to develop a model to relate the electrical input to the droplet position. Finally, the position of the droplet is measured as a function of the duty cycle of the input to verify the prediction of the idealized model to quantify the accuracy of the position model and to assess the potential accuracy/repeatability of the approach.

EWOD response is usually characterized by measuring the contact angle of a sessile droplet grounded by a fine wire. For a grounded droplet, the relationship between the applied voltage $(V)$ and the force per unit contact line $(f)$ can be expressed by the Young - Lippmann equation for EWOD $[25,26,27]$ :

$$
f=\left[\operatorname{Cos}\left(\theta_{E W}\right)-\operatorname{Cos}\left(\theta_{0}\right)\right] * \gamma=\frac{\varepsilon \varepsilon_{0}}{2 d} V^{2}=\frac{1}{2} c V^{2}
$$

where $\theta_{E W}, \theta_{0}$ are the contact angles with and without the applied voltage, $\varepsilon$ is the relative permittivity, $\varepsilon_{0}$ the permittivity of free space, $d$ the dielectric thickness, and $\gamma$ the interfacial tension between the droplet and the ambient. This can also be expressed simply in terms of the capacitance per unit area $(c)$. For a DC power supply, $V$ is simply the voltage from the power source. For an AC supply, the root mean square (RMS) voltage is used.

Anodizing electrodes have been previously used to increase the reliability of EWOD devices because the oxide heals dielectric defects $[24,27 \underline{25}, 28,29]$. In the present case coplanar electrodes are uniformly coated with a leaky dielectric over a passivating electrode. Electrically, this system is best modeled as two diodes in series similar to prior work with DC voltages $[12,23,24,25]$. This work considers the impact of AC inputs. The illustration in Figure 1 shows the system configuration, its equivalent circuit representation, and the resulting voltage across each electrode. When a voltage potential is applied, the majority of the voltage drop occurs across the reverse-biased diode so that the actuation force acts towards the higher potential. One side of the droplet is always reverse biased—reducing the current and resulting electrolysis. 

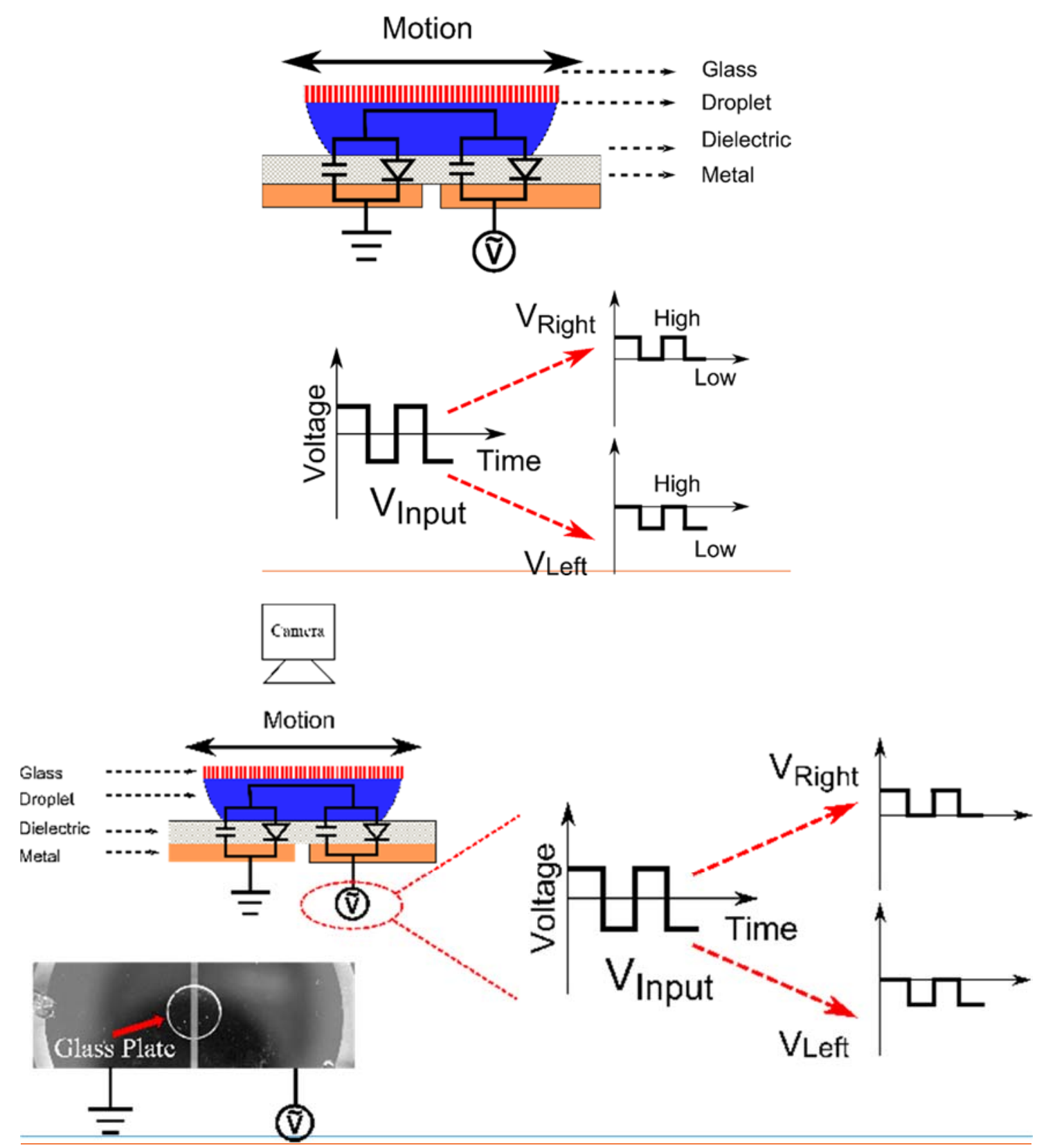


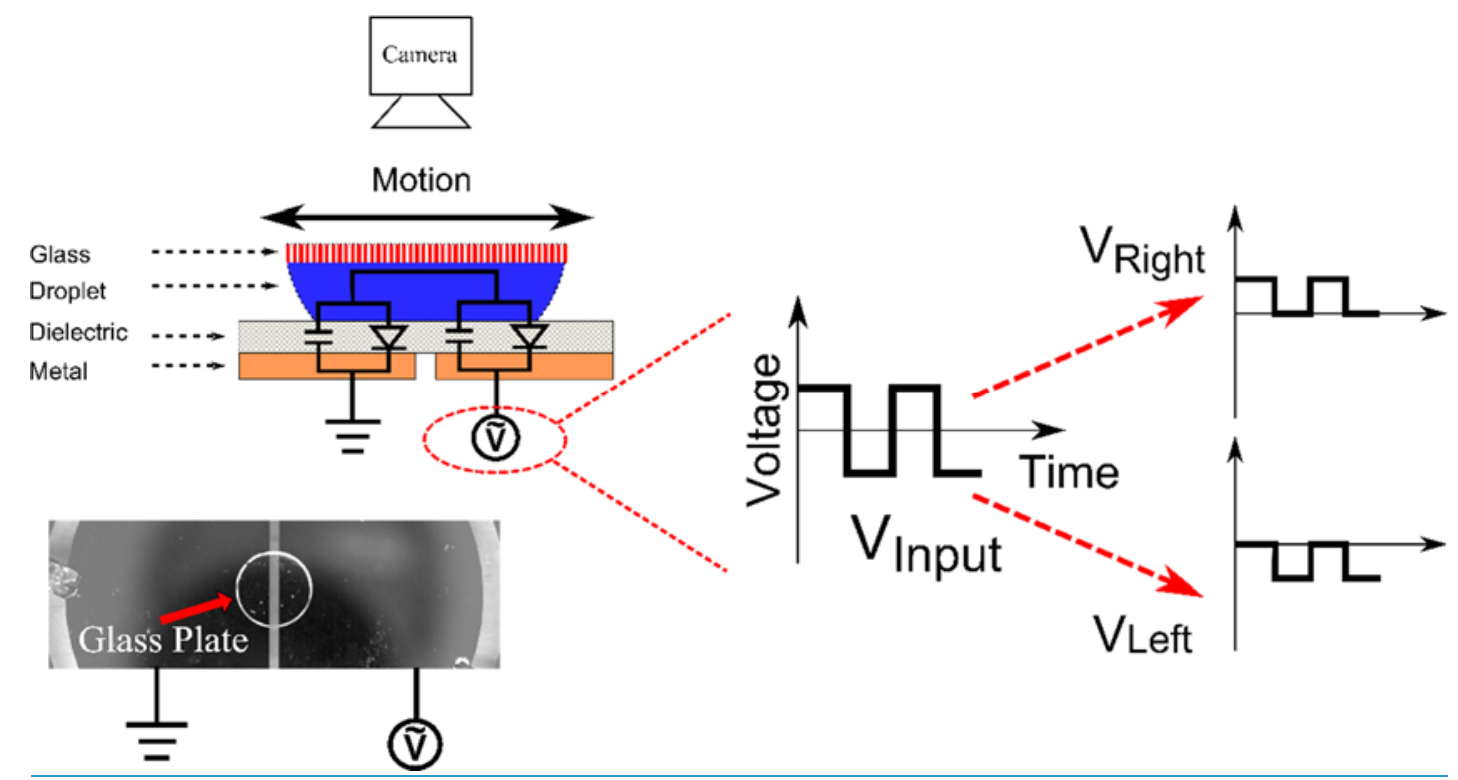

Figure 1: Proposed actuation scheme and its testing arrangement. Top left: Side view of the plate and camera for position measurement. The equivalent electrical circuit representation is overlaid on the actuator. A circular glass plate is carried by the EWOD actuated droplet and an $\mathrm{AC}$ square wave input is used $\left(\mathrm{V}_{\text {Input }}\right)$. TheRight: the voltage drop across the dielectric layer on the left and the right side of the droplet, the voltage -is $V_{\text {Left }}$ and $V_{\text {Right }}$, respectively. Bottom left: Sample image from the overhead camera. The diameter of the glass plate is $8 \mathrm{~mm}$.

For a symmetric square wave input, the voltage drop across the dielectric might be expected to follow the illustration shown in Figure 1. Most of the applied voltage drop appears on the higher potential side of the droplet/dielectric interface. The side with the larger voltage drop switches with the voltage polarity.

The force per unit length contact line acting on the droplet interface can be expressed using Equation 1:

$f=\frac{\varepsilon \varepsilon_{0}}{2 d} \tilde{V}_{\text {Right }}^{2}-\frac{\varepsilon \varepsilon_{0}}{2 d} \tilde{V}_{\text {Left }}^{2}=\frac{c}{2}\left(\tilde{V}_{\text {Right }}^{2}-\tilde{V}_{\text {Left }}^{2}\right)$

Equation 2

In Equation 2, the voltages $\tilde{V}_{R i g h t}^{2}$ and $\tilde{V}_{L e f t}^{2}$ are the RMS voltages across the dielectric at the right and left side of the droplet and the negative sign indicates the difference of the force direction.

For a square waveform, the RMS voltage can be expressed in terms of its duty cycle input. The duty cycle (D) of a square wave is defined as the ratio of the high pulse time $\left(t_{1}\right)$ over the period $(T)$. The time-averaged force over one period is then: 


$$
\begin{aligned}
f_{\text {net }}=\frac{c}{2} *\left(\frac{1}{T} \int_{0}^{t_{1}}\left(V_{R}^{2}(t)-V_{L}^{2}(t)\right) * d t+\frac{1}{T} \int_{t_{1}}^{T}\left(V_{R}^{2}(t)-V_{L}^{2}(t)\right) * d t\right) \\
=\frac{c}{2} *\left[\left(V_{R(\text { high })}^{2}-V_{L(\text { high })}^{2}\right) * D+\left(V_{R(\text { low })}^{2}-V_{L(\text { low })}^{2}\right) *(1-D)\right]
\end{aligned}
$$

where the subscripts high and low indicate the respective voltages at the left and right side of the droplet when the input is positive (high) or negative (low). Equation 3 predicts the time-averaged actuation force from the instantaneous voltage. However, it is very difficult to measure the voltage behavior due to the dynamics of the system and when the droplet is not centered the voltage across the dielectric will change because the capacitance of each side will be different.. To better understand the voltage response of the EWOD system, Matlab SimElectronics ${ }^{\circledR}$ was used with ideal elements to show the voltage variation of the circuit. The droplet position and input duty cycle relationrelations were developed using the simulation results. These are then compared to experimental position measurements.

\section{Electrical Model and Simulation}

Matlab SimElectronics ${ }^{\circledR}$ was used to simulate the idealized circuit behavior using an idealized diode model (piece-wise linear diode with $0.1 \mathrm{~V}$ turn-on voltage) and neglecting resistance in the system. The input voltage was set at $1000 \mathrm{~Hz}$ and the duty cycle of the input was varied. The effect of droplet position was simulated by varying the capacitances of the left and right capacitor while keeping the total capacitance $\left(C_{\text {Left }}+C_{\text {Right }}\right)$ fixed at $1 \mathrm{nF}$. This models the case of a droplet constrained by wetting to a plate to maintain a constant area. These conditions are met when the droplet height and gap between the electrodes are small relative to the width of the plate... This condition is adequate for the center regions, but may breakdown at extreme positions. 


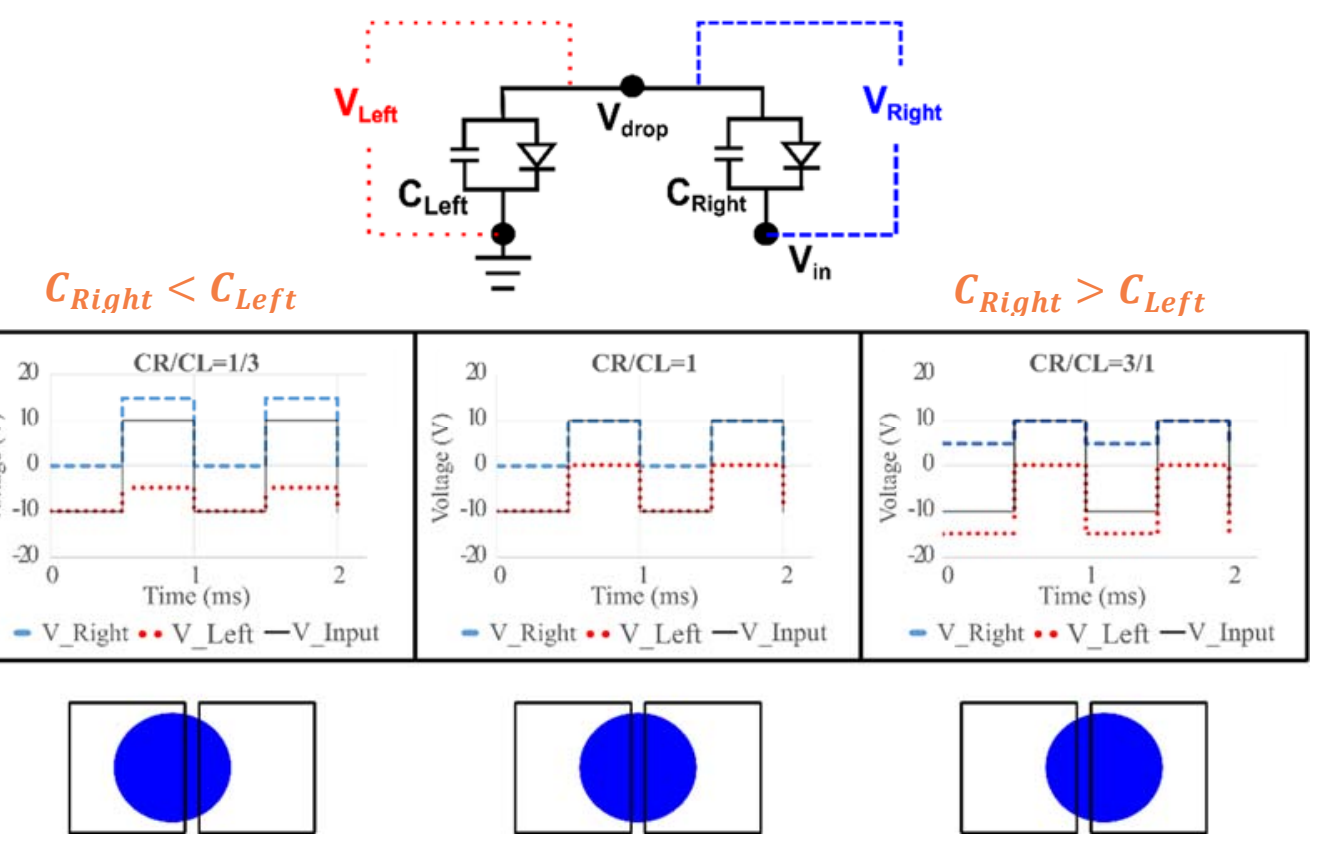

Figure 2: Top: the circuit representation of the co-planer electrowetting droplet with idealized diodes. $\mathrm{V}_{\text {drop }}$ is the droplet voltage. Middle:The voltage response of the simulated circuit when the capacitance was varied. The supplied signal was a $50 \%$ duty cycle square wave at $1000 \mathrm{~Hz},-10 \mathrm{~V}$ to $10 \mathrm{~V}$. The total capacitance is $1 \mathrm{nF}$. Bottom: the corresponding droplet positions at given capacitor ratio. With a 50\% duty cycle, the droplets are only stable in the middle position..

Figure 2 shows the circuit setup and the voltage variation across the interface at $50 \%$ duty cycle for three different droplet positions (capacitance ratios). For the idealized circuit, the peak voltages were only a function of the capacitance ratio between the two capacitors, regardless of the duty cycle input. When the capacitances were not equal between the left and right side $\left(C_{\text {Left }} \neq C_{\text {Right }}\right)$, the capacitor with larger capacitance would drive the voltage of the smaller capacitor above the input voltage. Both didoesdiodes prevent current conduction in steady-state and the droplet acquires a net charge $\left(\mathrm{V}_{\mathrm{drop}} \neq 0\right)$. The value of the respective voltages as a function of the input voltage and the capacitances of the two capacitors are listed in Table 1.

Table 1: Simulated voltages as a function of the capacitance of the left and right capacitors. $V_{\text {in }}$ is the input voltage, High is when the input is positive and low is when the input is negative. $V_{\text {Right }}$ and $V_{\text {Left }}$ are the voltages across the dielectric at the left and right side of the droplet, respectively.

\begin{tabular}{|c|c|c|c|c|}
\hline & \multicolumn{2}{|c|}{$C_{\text {Right }}>C_{\text {teft }} C_{\text {Right }}<C_{\text {Left }}$} & \multicolumn{2}{|c|}{$C_{\text {Right }}<C_{\text {teft }} C_{\text {Right }}>C_{\text {Left }}$} \\
\hline & $V_{\text {right }}$ & $V_{\text {Left }}$ & $V_{\text {Right }}$ & $V_{\text {Left }}$ \\
\hline $\begin{array}{c}\text { High } \\
\left(\mathrm{V}=+V_{\text {in }}\right)\end{array}$ & $\begin{array}{l}\frac{2 * C_{\text {Left }}}{C_{\text {Right }}+C_{\text {Left }}} \\
*\left|V_{\text {in }}\right|\left|V_{\text {in }}\right|\end{array}$ & $\frac{C_{\text {Right }}-C_{\text {Left }}}{C_{\text {Right }}+C_{\text {Left }}} *\left|V_{\text {in }}\right| \theta$ & $\begin{array}{l}\left|V_{\text {in }}\right| \frac{2 * C_{\text {Left }}}{C_{\text {Right }}+C_{\text {Left }}} \\
*\left|V_{\text {in }}\right|\end{array}$ & 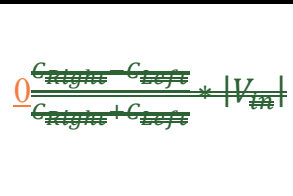 \\
\hline
\end{tabular}




\begin{tabular}{|c|c|c|c|c|}
\hline $\begin{array}{c}\text { Low } \\
\left(V=-V_{\text {in }}\right)\end{array}$ & 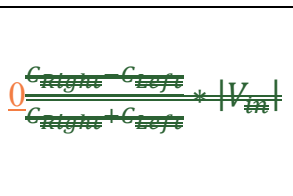 & $\begin{array}{l}-\left|V_{\text {in }}\right| \frac{-2 * C_{\text {Right }}}{C_{\text {Right }}+C_{\text {Left }}} \\
*\left|V_{\text {in }}\right|\end{array}$ & $\frac{C_{\text {Right }}-C_{\text {Left }}}{C_{\text {Right }}+C_{\text {Left }}} *\left|V_{\text {in }}\right| \theta$ & $\begin{aligned} \frac{-2 * C_{\text {Right }}}{C_{\text {Right }}+C_{\text {Left }}} * & \left|V_{\text {in }}\right| \\
& =\left|V_{i n}\right|\end{aligned}$ \\
\hline
\end{tabular}

The area of the circular droplet covering the right and the left electrodes is defined in terms of total area ratio $\left(A_{\text {right }}=\right.$ $R_{a} * A_{\text {Total }}$, and $\left.A_{\text {Left }}=\left(1-R_{a}\right) * A_{\text {Total }}\right)$. For a uniform dielectric layer, the capacitance can also be expressed using the area ratio $\left(R_{a}\right)$ since $\mathrm{C}=\frac{\varepsilon}{d} * A$. The time-averaged net force is found by integrating the force over a full period. By varying the relative time that the positive and negative voltages are applied (duty cycle), a point can be found where the droplet is in static equilibrium for a given area ratio. Higher voltages (and forces) on one side are offset by a shorter time over which the voltage is applied.

Rewriting Equation 3 in terms of the area ratio,

$$
\begin{aligned}
& f_{\text {net }}\left(R_{a}, D\right) \\
& =\left\{\begin{array}{rr}
\frac{c}{2} *\left[\left(\left(2\left(1-R_{a}\right) V_{\text {in }}\right)^{2}-\left(\left(2 * R_{a}-1\right) V_{\text {in }}\right)^{2}\right) * D+\left(0-V_{\text {in }}^{2}\right) *(1-D)\right], & 0<R_{a}<0.5 \\
\frac{c}{2} *\left[\left(V_{\text {in }}^{2} * D\right)+\left(\left(\left(2 * R_{a}-1\right) V_{\text {in }}\right)^{2}-\left(-2 * R_{a} * V_{\text {in }}\right)^{2}\right) *(1-D)\right], & 0.5 \leq R_{a}<1
\end{array}\right. \\
& =\left\{\begin{array}{rr}
\frac{c}{2} V_{\text {in }}^{2} *\left[4 D\left(1-R_{a}\right)-1\right], & 0<R_{a}<0.5 \\
\frac{c}{2} V_{\text {in }}^{2} *\left[4 * R_{a} *(D-1)+1\right], & 0.5 \leq R_{a}<1
\end{array}\right.
\end{aligned}
$$

Finally, by solving for the area ratio where the net force is zero $\left(f_{\text {net }}=0\right)$ as a function of duty cycle, the relationship between the input duty cycle and the area ratio $\left(R_{e q}\right)$ at which the droplet will be in static equilibrium for the given duty cycle is:

$$
R_{e q}(D)=\left\{\begin{array}{cc}
1-\frac{1}{4 D}, & 0<D<0.5 \\
\frac{-1}{4(D-1)}, & 0.5 \leq D<1
\end{array}\right.
$$

Equation 5

The relationship between the area ratio and the equilibrium position depends on the shape of a droplet. This work will consider the case of a droplet constrained to a circular shape which generates a nonlinear relationship between position and area ratio. 
For a rectangular shape, the area ratio would be proportional to linear position. When a droplet is wetting a solid object, the droplets can be constrained to a circle or even a square shape by patterning the wetted region to match the desired droplet shape and maintaining a "thin" droplet [29].[30]. Thus both shapes are relevant to practical actuation tasks.

Equation 4 predicts that the actuating force is linearly dependent on the area ratio. The magnitude of the restoring force increases as the droplet's position moves away from equilibrium. For the idealized case, the input duty cycle is limited from $25 \%-75 \%$ as any input beyond the range will result in a non-physical value of the area ratio required for static equilibrium $\left(\mathrm{R}_{\mathrm{a}}<0\right.$ or $\left.\mathrm{R}_{\mathrm{a}}>1\right)$.

The diodes are required for this actuation mode. With the diodes removed, the droplet would be floating and the droplet would always be in equilibrium with equal areas on both electrodes regardless of the input waveform [13] because the actuation force would not be polarity dependent without the diodes.

\section{Model Validation}

To test these predictions, a circular plates werewas placed on a $50 \mu \mathrm{L}$-droplets droplet and the position of the plate was measured as a function of the duty cycle of the square wave input. Signal voltages varied from $+V_{\max }$ to $-V_{\max }$. Sample substrates were prepared by photolithographically patterning $400 \mathrm{~nm}$ titanium which was evaporated onto $75 \mathrm{~mm}$ x $50 \mathrm{~mm}$ clean glass slides $\left(\right.$ Corning $\left.^{\circledR}\right)$. After patterning, dilute Cytop ${ }^{\circledR} 809 \mathrm{M}$ ( $2: 1 \div 2$ or $\underline{1: 2: 1}$ solution to solvent ratio by weight) was spin-coated and annealed according to manufacturer's specifications. The resulting Cytop ${ }^{\circledR}$ thickness was $380280 \pm 10 \mathrm{~nm}$ and $100 \pm 10 \mathrm{~nm}$, respectively. The thickness of the coating was measured by Rudolph ellipsometer and verified by Alfa step profilometer.

The metal film was patterned with a $300 \mu \mathrm{m}$ gap separating the left and right electrodes. The droplets weredroplet used was $0.1 \mathrm{M}$ citric acid droplet aqueous solution with OS-30 silicone oil ambient. A circular glass plate (diameter $8 \mathrm{~mm}$, thickness $0.1 \mathrm{~mm}$ ) was carried by the $50 \mu \mathrm{L}$ droplet. The strong wetting between the droplet and the glass top plate constrained the droplet to remain circular during motion. Electrical signal was generated using a digital function generator (Agilent 33250A) and amplified (Kepco BOP 100-1M). The desired frequency $(1000 \mathrm{~Hz})$ was set with the initial duty cycle at $50 \%$ to position the droplet/plate at the boundarycenter between two electrodes-(see Figure 1). Initial tests used $380280 \mathrm{~nm}$ Cytop coating.- The driving voltage ( $\pm 30 \mathrm{~V}$, peak to peak) was chosen because it was the largest voltage without significant electrolysis. AfterFor voltages at or below 30 volts, no performance change was observed over the voltage was switched oncourse of the testing. When the input signal was applied for 40 minutes $\left(30 \mathrm{~V}_{\mathrm{pp}}, 1 \mathrm{kHz}\right.$ and $50 \%$ duty cycle), stepping actuation was still observed when the duty cycle was changed. At voltages above 30 volts, the electrochemical reaction reduced the reliability of the device due to the gas bubbles generated under the plate. To ensure a consistent starting conditions, the duty cycle was switched from 
high (70\%) to low (30\%) then back for several cycles after the initial voltage application to eliminate the effect of contact angle hysteresis. Finally, the duty cycle was adjustedThe same procedure was carried out for new substrates only. The data was then collected by adjusting the duty cycle to the full opposite (between 30\% to 70\%) of the desired value then back. For example, if the final value of the input was $40 \%$, the input would go $70 \%$ then $40 \%$; if the desired final input was $65 \%$, the input would go $30 \%$ then $65 \%$. One complete cycle (position corresponding to duty cycle from $30 \%$ to $70 \%$ ) as recorded. This was repeated

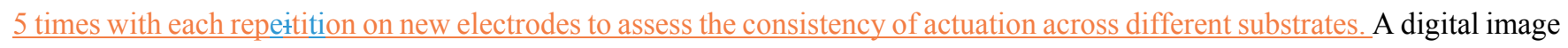
was taken from above to measure the resulting position. The captured images were then analyzed using Matlab® image analysis tool to find the position of the centroid of the glass plate.

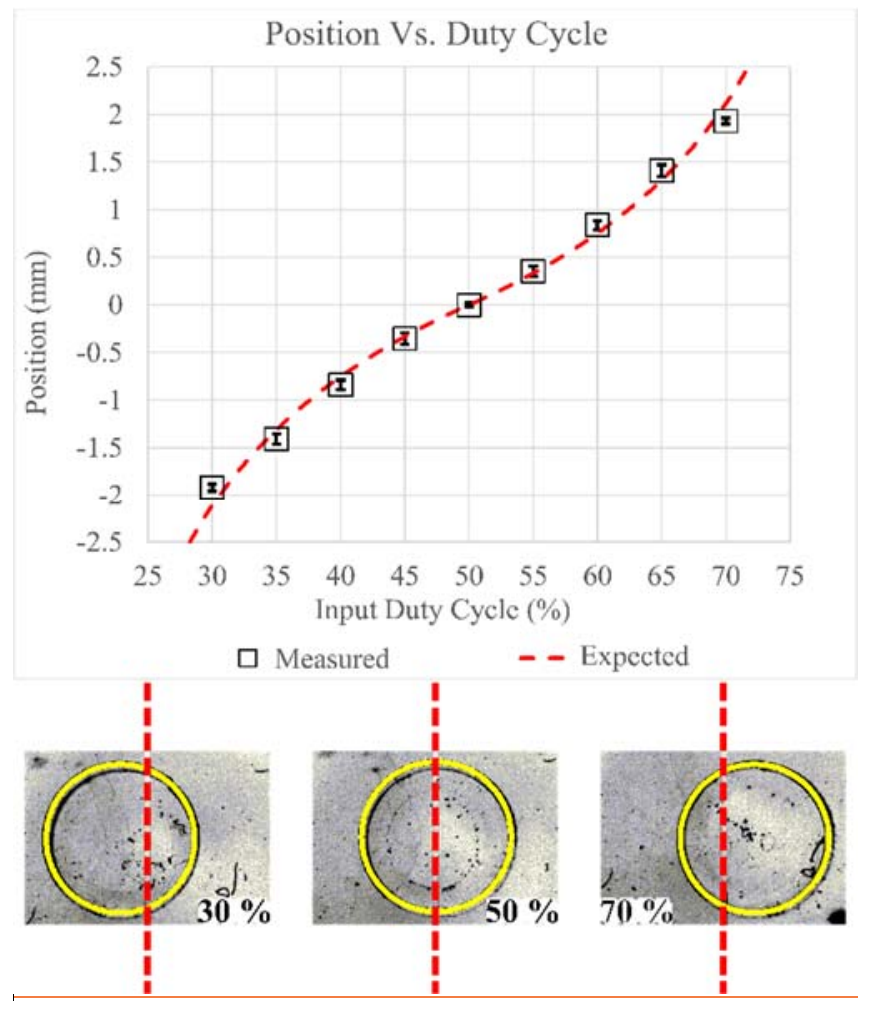

Figure 3: The measured plate position Vs. duty cycle input. Tests were done using maximum available step size at $30 \mathrm{~V}_{\mathrm{pp}}$. The squares represent the measured position of the plate centroid. The dashed line is the model prediction based on Equation 5. Five tests were performed for each data point and the standard deviation of the position was reported. The bottom row is the images of the plates with the corresponding duty cycle input-after image processing tool found the boundary of the plate. For easier viewing, the colors of the images are inverted. The dark line in the middle of the image is the $300 \mu \mathrm{m}$ gap between the electrodes. The position is measured from the centroid of the plate to the centerline between the two electrodes. 
Figure 3Figure 3Figure 3 compares the measured relationship between the position of the plate and the duty cycle of the input voltage and the analytical predictions using idealized diodes. Five tests were performed for each data point and the error bars represent the standard deviation of the results. Using Equation 5, the equilibrium area ratio $R_{e q}$ was related to the centroid of the circle (accounting for the $300 \mu \mathrm{m}$ gap) and the predicted centroid position from Equation 5 is plotted against the measured positions. As predicted, the droplet/plate successfully switches between multiple equilibrium positions by changing the input square wave's duty cycle with very repeatable results with a maximum standard deviation of $0.063 \mathrm{~mm}$. While the model based on idealized linear diodes fit the experimental data fairly well, there are deviations — especially at the extreme positions.

One source of the position error may be difference between the contact area of the droplet and the plate. The model is based on the droplet position while the experiments measured the plate position. In the oil ambient, the droplet contact area is smaller than the plate diameter and the deviation could become significant at the extreme positions. To further improve the positioning at the ends, the droplet contact area could be tracked or a contact angle between the droplet and substrate close to $90^{\circ}$ could be used. The other significant error source may be in the assumption of idealized diodes. Real diodes have leakage current which would slow the response of the diodes and would cause larger deviations at the extreme positions. Circuit simulations suggest that this is the largest source of deviation from the idealized model. This could be accounted for with an improved model (theoretical or empirical).

\section{Time Response}

The response time of the droplet/plate was also investigated by applying a step change in the input duty cycle at time $>0$, the plate position was tracked optically at 50 frames/second from above. Duty Cycle steps of $10 \%, 20 \%, 30 \%$ and $40 \%$ were tested. A total of 5 tests were done for each step size and the resulting average position vs. time information are plotted in Figure 4 (left). 


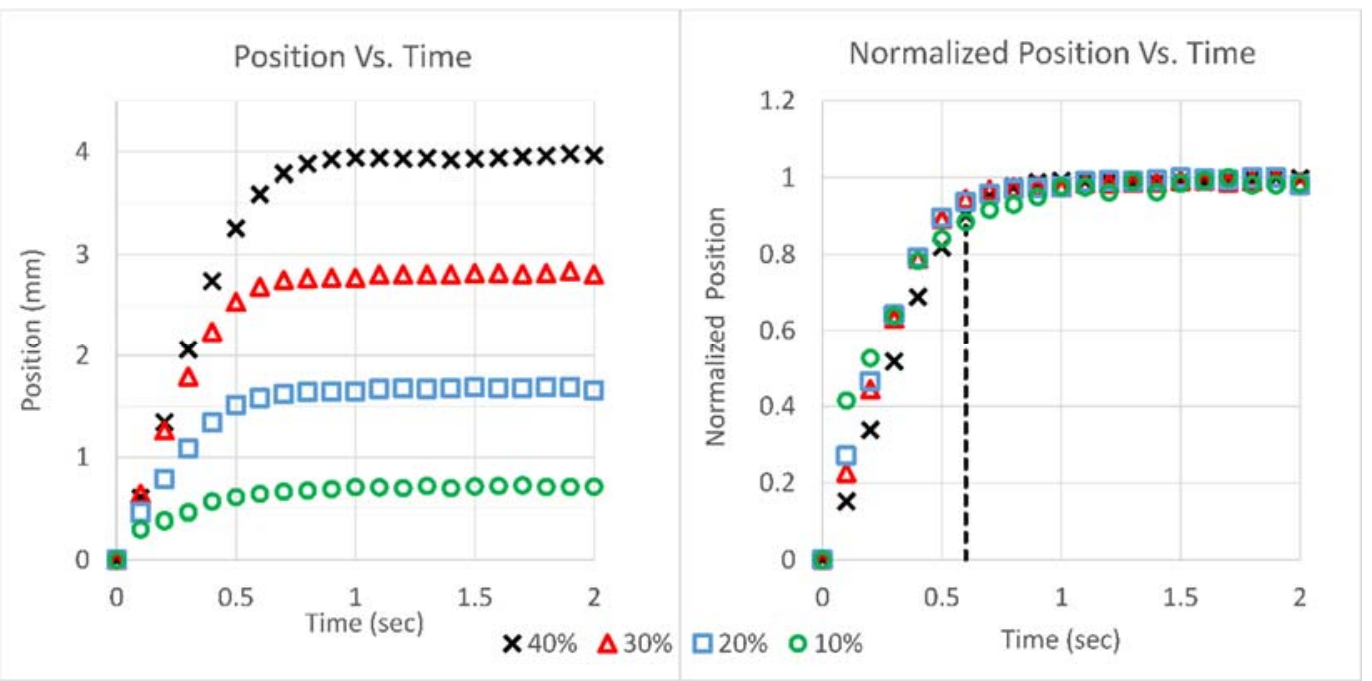

Figure 4: Left: the position vs. time plot of the actuated plate under different step changes in duty cycle. A total of 5 tests were done for each set of data, the average values are plotted. The standard deviations of the positions were between 0.04 and $.012 \mathrm{~mm}$ (smaller than the markers used). Right: the graph shows the normalized position (dividing the position by its steady state location) vs. time in seconds. All tested cases reached $90 \%$ of steady state positions in less than 0.6 second.

Figure 4 shows the response in both distance and normalized by the steady state position. The characteristic time for the plate to reach $90 \%$ of the steady state position was found to be less than 0.6 second for the voltage tested. No overshoot was observed. When the step change is applied, the restoring force would be at its maximum. As the droplet moves closer to its final position, the force rapidly decays due to the voltage shift caused by the capacitance change. Dissipative forces such as hydrodynamic shear (both within the droplet and the medium).) and contact line friction are significant. The combined effects result in a "soft landing" effect which could serve as an additional bonus in moving delicate components.

\section{Accuracy and Repeatability}

To evaluate the relationship of the droplet position to the step size, another series of experiments were performed. The droplet/plate was set at the center first by applying a 50\% duty cycle, then the input duty cycle was ramped up to $70 \%$ down to $30 \%$ and then back to $50 \%$. The duty cycles waswere ramped in fixed steps of $10 \%$ every 2 seconds and corresponding position tofor each input was recorded. All test were repeated 5 times; each in a new location on the electrodes with a new droplet. The These results were compared to the previous experiments measuring position using the maximum available-step size were compared to the positions when steppedtests in inerements of $10 \%$ which the droplet was moved to the farthest duty cycle (in the range $30-70 \%$ ) and then to the target position. The maximum deviation from the idealized electric circuit model predictions from Equation 5 is presented as the accuracy of the system for each condition. The standard deviation in positioningof the 
position was calculated for each duty cycle input and the average of the standard deviations across all positions is presented as the bidirectional repeatability. For unidirectional repeatability, the standard deviations of the position are calculated separately for increasing and decreasing duty cycles and the maximum value reported. The results are listed in Table 2 , together with the results from the maximum step size tests.

Since lower voltage is preferred for many applications, a thinner $(100 \mathrm{~nm} \pm 10 \mathrm{~nm})$ dielectric for lower actuation voltage was also investigated, the thickness of the dielectric.. A $10 \mathrm{~V}_{\mathrm{pp}}$ input voltage was used as it was the largest that avoided electrolysis. The above tests were repeated and the positioning accuracy and repeatability were calculated for comparison to the tests with the $300280 \mathrm{~nm}$ dielectric. The data are summarized in Table 2. At the lower voltage (thinner dielectric), the repeatability deteriorated significantly which suggests that fluid friction and other losses are significant relative to the actuation force. Although the unidirectional repeatability did not improve significantly with step size for either sample type, the higher voltage during actuation did significantly reduce the variation. The large difference between bidirectional and unidirectional repeatability is likely due to actuation losses from viscous drag and contact line friction. The average deviation from the prediction (Equation 5) was $0.106 \mathrm{~mm}$ (2.3\% of the plate diameter). The accuracy of the system model could be improved by utilizing a more accurate circuit model or by substituting an empirical relationship for Equation 5 . The best repeatability was obtained by using maximum step size and a thicker dielectric, the resulting value was $0.063 \mathrm{~mm}(0.8 \%$ of the plate diameter).

Table 2: The accuracy and the repeatability of the actuation system as a function of the step size

\begin{tabular}{|c|c|c|c|c|}
\hline & \multicolumn{2}{|c|}{$30 \mathrm{~V}_{\mathrm{pp}} / 300 \underline{\underline{2} 80} \mathrm{~nm}$ thick Cytop } & \multicolumn{2}{c|}{$10 \mathrm{~V}_{\mathrm{pp}} / 100 \mathrm{~nm}$ Cytop } \\
\hline & $10 \%$ Step Size & Max Step & $10 \%$ Step Size & Max Step \\
\hline Accuracy (mm) & 0.181 & 0.181 & 0.293 & 0.191 \\
\hline $\begin{array}{c}\text { Bidirectional } \\
\text { Repeatability (mm) }\end{array}$ & 0.153 & N.A. & 0.204 & N.A. \\
\hline $\begin{array}{c}\text { Unidirectional } \\
\text { Repeatability (mm) }\end{array}$ & 0.08 & 0.063 & 0.189 & 0.182 \\
\hline
\end{tabular}

Comparing the electrowetting number $\left(\eta=\frac{\varepsilon \varepsilon_{0}}{2 \gamma} * \frac{V^{2}}{d}\right)$ at the testing voltages, the ratio of their respective electrowetting number is $3: 1$ between the thicker coating $(300 \underline{280} \mathrm{~nm} / 30 \mathrm{~V})$ and thinner coating $\left(100 \mathrm{~nm} / 10 \mathrm{~V}_{\mathrm{pp}}\right)$. Thus, the actuation force should be three times higher for the thick coating/higher voltage combination since the actuation force is proportional to the 
electrowetting number. On the other hand, contact angle hysteresis which is the difference between the advancing contact and the receding contact angles or the energy lost per unit contact line [30][31], can remain fairly constant under EWOD actuation [31].[32]. The higher electrowetting number/actuation force improved repeatability due to the higher applied force relative to the dissipative forces.

Since the actuation force and contact line friction are both proportional to the plate diameter, it is expected that the accuracy and repeatability could scale linearly with the plate/droplet diameter. Smaller plates, should achieve higher positioning accuracy. This would require a decrease in droplet volume as well to maintain a similar droplet aspect ratio (height/diameter) for plate stability. A surface texture could be introduced to decrease contact angle hysteresis, but may also reduce the electrowetting actuation force due to the reduced droplet/substrate contact area [3233]. Li and Mugele have shown that AC electrowetting inputs can significantly decrease the contact line friction effects [3334]. It is possible that the contact line friction effects could be reduced by selecting the actuation frequency to maximize this effect. WhileThe actuation force is proportional to the distance between the droplet and the equilibrium position. Small steps in duty cycle may not adequately overcome dissipative forces such as contact line friction and viscous drag. Experiments showed that duty cycle steps below 5\% did not show consistent actuation, suggesting that the actuation force was comparable to the dissipative forces under these test conditions. However, since the applied duty cycle is continuously variable-so that any plate position between the extreme testing positions can still be achieved using this actuation method. A small movement ( $<10 \%$ duty cycle) could be achieved using this arrangementby first moving the droplet farther away from the desired position before inputting the target duty cycle.

\section{The maximum step size data is an implementation of this approach.}

\section{Conclusion}

This work describes a novel actuation/control scheme using electrowetting on dielectric with coplanar electrodes. Utilizing the unique diode-like properties of the electrolyte/dielectric/metal combination, an AC square wave was used to move a droplet carrying a glass plate in steps that arewere a fraction of the electrode size. Unlike conventional EWOD driven droplet transportation; multiple stable positions within the electrodes eancould be reached by adjusting the duty cycle of the input square wave.

An electrical model was simulated using idealized electrical elements. The relation between the input duty cycle and the responding droplet position was developed based on the simulation results. These results show that the position can be controlled through the central region of the electrode width, but the idealized model predicts that no duty cycle achieves a stable position on the extreme edges of the electrodes. The idealized relation matches measured plate positions to within $2.5 \%$ of the 
droplet diameter and repeatability of the actuation system was $0.8 \%$ of the droplet diameter for large steps. This is a substantial improvement in droplet control compared to existing close-loop methods that achieve steps that are $20 \%$ of the droplet size.

The repeatability is shown to decrease when taking smaller steps or when actuated at lower voltages. The error is attributed to the contact line friction in the system. The accuracy of the system model could be improved by using a refined circuit model based on non-ideal diodes. These results could also be improved through reduction of contact line friction. This is a substantial improvement in droplet control compared to existing close-loop methods that achieve steps that re only $20 \%$ of the droplet size.The repeatability and minimum step size could also be improved through reduction of contact line friction. . The system described here could also be adapted for long range motinousing low frequency with asymmetric electrode designs as demonstrated by Fan et al. [23] while maintaining the capability for high resolution, sub-electrode positioning through high

\section{frequency inputs.}

In conclusion, by moving the droplet sub-electrode in a deterministic manner, the proposed control scheme could improve the positioning accuracy of the droplet while reduce the electrical wiring complexity in electrowetting systems. A stepped actuation with DC voltage could be used for large motion and fine positioning accomplished by varying the duty cycle in an AC input. Potentially, this approach could be used to fabricate a droplet-based actuator/positioner to provide a new alternative for compact millimeter scale actuators which can travel large distance while maintain the positioning accuracy without the need to incorporate location sensing and feedback control-

\section{Acknowledgments}

This work is supported in part by the National Science Foundation (NSF) under grant No. CMMI-1130751130755

\section{References}

[1] D.J. Bell, T.J. Lu, N.A. Fleck, S.M. Spearing, MEMS actuators and sensors: observations on their performance and selection for purpose, J Micromech Microeng, 15(2005) S153-S64.

[2] N.B. Hubbard, M.L. Culpepper, L.L. Howell, Actuators for micropositioners and nanopositioners, Applied Mechanics Reviews, 59(2006) 324-34.

[3] P. Lambert, U.1.d.B.C. 165-14, pierre.lambert@ulb.ac.be, F. Seigneur, E.P.F.d. Lausanne, frank.seigneur@epfl.ch, et al., Design of a Capillary Gripper for a Submillimetric Application, IPAS, Springer US2016, pp. 3-10.

[4] M. Mastrangeli, The Fluid Joint: The Soft Spot of Micro- and Nanosystems, Adv Mater, 27(2015) 4254-72. 
[5] L. Jiang, D. Erickson, Directed Self-Assembly of Microcomponents Enabled by Laser-Activated Bubble Latching, Langmuir : the ACS journal of surfaces and colloids, 27(2011) 11259-64.

[6] R.R.A. Syms, E.M. Yeatman, V.M. Bright, G.M. Whitesides, Surface tension-powered self-assembly of microstructures - The state-of-the-art, Journal of Microelectromechanical Systems, 12(2003) 387-417.

[7] N.B. Crane, O. Onen, J. Carballo, Q. Ni, R. Guldiken, Fluidic assembly at the microscale: progress and prospects, Microfluidics and Nanofluidics, 14(2012) 383-419.

[8] R. Shamai, D. Andelman, B. Berge, R. Hayes, Water, electricity, and between... On electrowetting and its applications, Soft Matter, 4(2008) 38-45.

[9] C.R. Knospe, S.A. Nezamoddini, Capillary force actuation, Journal of Micro-Nano Mechatronics, 5(2010) 57-68.

[10] J. Lee, C.J. Kim, Surface-tension-driven microactuation based on continuous electrowetting, Journal of Microelectromechanical Systems, 9(2000) 171-80.

[11] A. Takei, K. Matsumoto, I. Shomoyama, Capillary motor driven by electrowetting., Lab on a chip, 10(2010) 1781-6.

[12] C.W. Nelson, C.M. Lynch, N.B. Crane, Continuous electrowetting via electrochemical diodes, Lab on a chip, 11(2011) 2149-52.

[13] I. Moon, J. Kim, Using EWOD (electrowetting-on-dielectric) actuation in a micro conveyor system, Sensor Actuat a-Phys, 130(2006) 537-44.

[14] G. Fantoni, H.N. Hansen, M. Santochi, A new capillary gripper for mini and micro parts, Cirp AnnManuf Techn, 62(2013) 17-20.

[15] J.H. Chang, J.J. Pak, Twin-plate electrowetting for efficient digital microfluidics, Sensor Actuat BChem, 160(2011) 1581-5.

[16] C.G. Cooney, C.Y. Chen, M.R. Emerling, A. Nadim, J.D. Sterling, Electrowetting droplet microfluidics on a single planar surface, Microfluidics and Nanofluidics, 2(2006) 435-46.

[17] M.G. Pollack, R.B. Fair, A.D. Shenderov, Electrowetting-based actuation of liquid droplets for microfluidic applications, Appl Phys Lett, 77(2000) 1725-6.

[18] S.K. Cho, H.J. Moon, C.J. Kim, Creating, transporting, cutting, and merging liquid droplets by electrowetting-based actuation for digital microfluidic circuits, Journal of Microelectromechanical Systems, 12(2003) 70-80.

[19] R.B. Fair, Digital microfluidics: is a true lab-on-a-chip possible?, Microfluidics and Nanofluidics, 3(2007) 245-81.

[20] E.J. Griffth, S. Akella, M.K. Goldberg, Performance Characterization of a Reconfigurable Planar Array Digital Microfluidic System, (2006) 329-56.

[21] J. Lee, H. Moon, J. Fowler, T. Schoellhammer, C.J. Kim, Electrowetting and electrowetting-ondielectric for microscale liquid handling, Sensor Actuat a-Phys, 95(2002) 259-68.

[22] J. Gong, C.J. Kim, All-electronic droplet generation on-chip with real-time feedback control for EWOD digital microfluidics, Lab on a chip, 8(2008) 898-906.

[23[23] S.K. Fan, H. Yang, T.T. Wang, W. Hsu, Asymmetric electrowetting--moving droplets by a square wave, Lab on a chip, 7(2007) 1330-5.

[24] N.B. Crane, A.A. Volinsky, P. Mishra, A. Rajgadkar, M. Khodayari, Bidirectional electrowetting actuation with voltage polarity dependence, Appl Phys Lett, 96(2010)-) 104103.

[2425] M. Khodayari, B. Hahne, N.B. Crane, Long Life Electrochemical Diodes for Continuous Electrowetting, J Electrochem Soc, 161(2014) E105-E11.

[2526] T.B. Jones, More about the electromechanics of electrowetting, Mechanics Research Communications, 36(2009) 2-9.

[2627] Q. Ni, D.E. Capecci, N.B. Crane, Electrowetting force and velocity dependence on fluid surface energy, Microfluidics and Nanofluidics, 19(2015) 181-9. 
[2728] M. Khodayari, J. Carballo, N.B. Crane, A material system for reliable low voltage anodic electrowetting, Mater Lett, 69(2012) 96-9.

[2829] M. Dhindsa, J. Heikenfeld, W. Weekamp, S. Kuiper, Electrowetting without electrolysis on selfhealing dielectrics, Langmuir : the ACS journal of surfaces and colloids, 27(2011) 5665-70.

[2930] N.B. Crane, P. Mishra, A.A. Volinsky, Characterization of electrowetting processes through force measurements, Rev Sci Instrum, 81(2010) 043902.

[3031] L. Gao, T.J. McCarthy, Contact angle hysteresis explained, Langmuir : the ACS journal of surfaces and colloids, 22(2006) 6234-7.

[3132] W.C. Nelson, P. Sen, C.J. Kim, Dynamic contact angles and hysteresis under electrowetting-ondielectric, Langmuir : the ACS journal of surfaces and colloids, 27(2011) 10319-26.

[3233] J. Heikenfeld, M. Dhindsa, Electrowetting on superhydrophobic surfaces: Present status and prospects, J Adhes Sci Technol, 22(2008) 319-34.

[3334] F. Li, F. Mugele, How to make sticky surfaces slippery: Contact angle hysteresis in electrowetting with alternating voltage, Appl Phys Lett, 92(2008). 
Author Biographies and Photos

Qi Ni

Qi Ni received his Bachelor of Science in Mechanical Engineering from University of South Florida (USF) in 2011. He is currently pursuing his Ph.D. in mechanical engineering at USF with a special focus on droplet-based mechanical actuators. His research interests include droplet manipulation using electrowetting on dielectric, directed interfacial assembly using fluid - structure interaction and droplet wetting with textured surfaces.

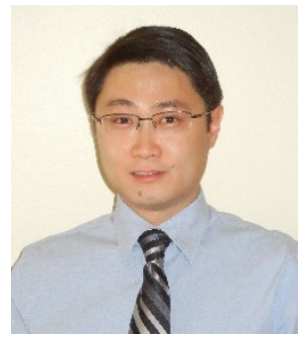

\section{Dan Capecci}

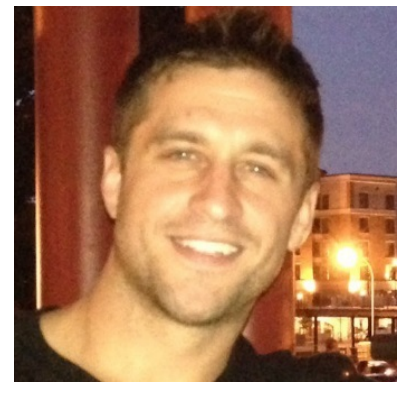

Daniel Capecci received his B.Sc in Mechanical Engineering from the University of South Florida in 2015. His current research interests include microfluidics, additive manufacturing, and haptics. He will begin pursuing his Ph.D. in 2016.

\section{Nathan Crane}

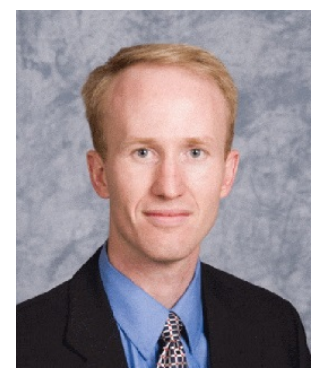


Nathan Crane received his B.S. and M.S. degrees in Mechanical Engineering at Brigham Young University in Provo, UT in 1998 and 1999 respectively. He completed a Ph.D. degree in Mechanical Engineering with a minor in Materials Science at the Massachusetts Institute of Technology in 2005. He worked for Caldera Engineering from 1998-1999 and with Pratt and Whitney Aircraft from 1999-2001. After completing his Ph.D., Dr. Crane worked at Sandia National Laboratories from 2005-2006. In 2006, Dr. Crane joined the University of South Florida where he is now an associate professor in the department of Mechanical Engineering. Dr. Crane's research interests lay in the areas of design and advanced manufacturing with a particular interest in additive manufacturing (3D Printing) and digital microfluidics. Recent projects have included microscale actuation using droplet microfluidics, capillary self-assembly process models, thermoelectric device design, and additive manufacturing of RF systems. His work has been recognized with the 2005 Solid Freeform Fabrication Symposium (SFF) Best Paper Award, and first place in the Graduate Student Mechanism Design Competition, a 2015 USF Outstanding Faculty Award. Dr. Crane was also a 2014 Fulbright Scholar. 